\title{
The construction of knowledge innovation platform and the analysis of data flow
}

\author{
Jinwen Li, Dingguo Sheng, Yanli Ma \\ Chinese North Engine Research Institute, Tianjin, 300400, China
}

Keywords: Knowledge field, Flow law, Knowledge flow, Knowledge accumulation.

\begin{abstract}
The existing knowledge management platform cannot well support the design and development of the large power diesel engine, based on the function and requirement 's analyses of the knowledge management on the innovation platform, through the introduction of the integrated delivery, forming an innovation platform architecture model of the effective integration of distributed knowledge resources and promoting the services' flow, finally putting forward the knowledge service model using $\mathrm{R} \& \mathrm{D}$ design flow, respectively, from the micro and macro aspects of knowledge flowing through comprehensive study. Analysing the knowledge system physiology from the energy and the process draws that these rules supporting the running of the platform is feasible.
\end{abstract}

\section{Introduction}

At present, the research in the knowledge flow of the knowledge management platform and services is a little and is mainly from the perspective of network collaborative design, such as the cooperative work is supporting the CEE geographical position by NASA, the purpose is to analyse the integration of multidisciplinary design and life cycle. [1]

\section{Research on the platform frame system and the key technology}

The design goal of the modern knowledge management service platform is aided design personnel in the knowledge resources massive, quickly finding the design available resources applied to the actual design process, in order to improve the design efficiency and quality. Considering the massive design knowledge resources distributed in the existing platforms, the dynamic response and support, platform scalability and the difficulty of the construction, the distributed interactive concept is accepted.

\section{Knowledge service integration mode based on design process}

Considering the contradiction covers the breadth and depth complexity, the design process of the actual design work, refining and defining the process of the knowledge flow is very difficult. This paper tries to describe the design process including the process of decomposition, the reorganization of knowledge unit and the knowledge service platform, realize the distributed resource environment.

\section{Architectural patterns and implementation method of the knowledge service platform}

In the face of the specific needs of microscopic changes and current research design, through the integration of knowledge to comb the theory and process planning, the knowledge service system needs deepening and improvement in the literature, fig 1. 


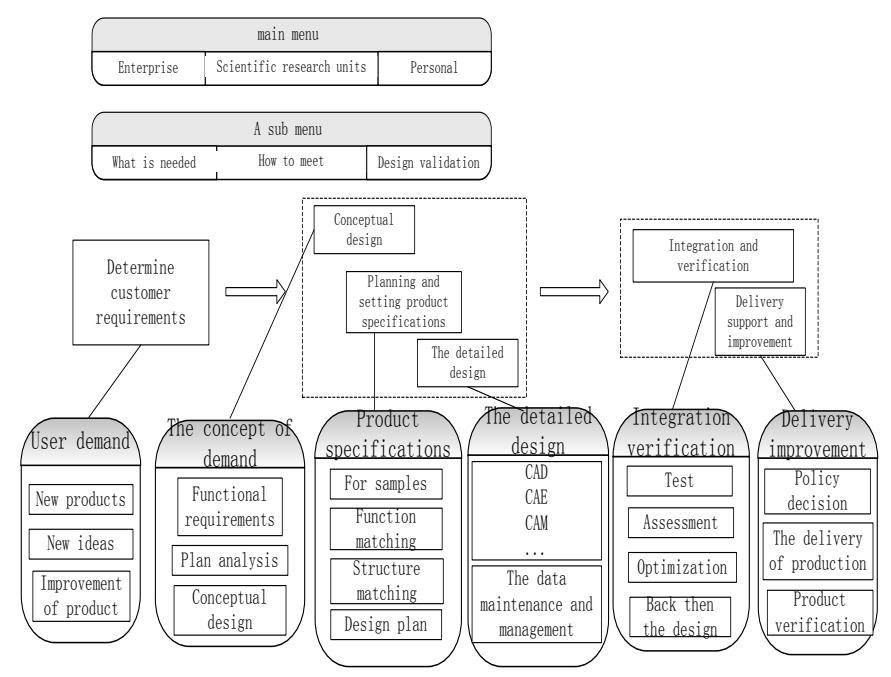

Fig 1 the knowledge service system

A feasible implementation scheme is introduced in this paper according to the thought of network technology and the open network service architecture (OGSA). Referring to the concrete research content and target the platform structure is designed. The whole framework of the knowledge service platform's modern design is proposed, as shown in Fig 2 consists of 4 layers. The integration of distributed network architecture is able to support the product design work in distributed resource environment. [2]

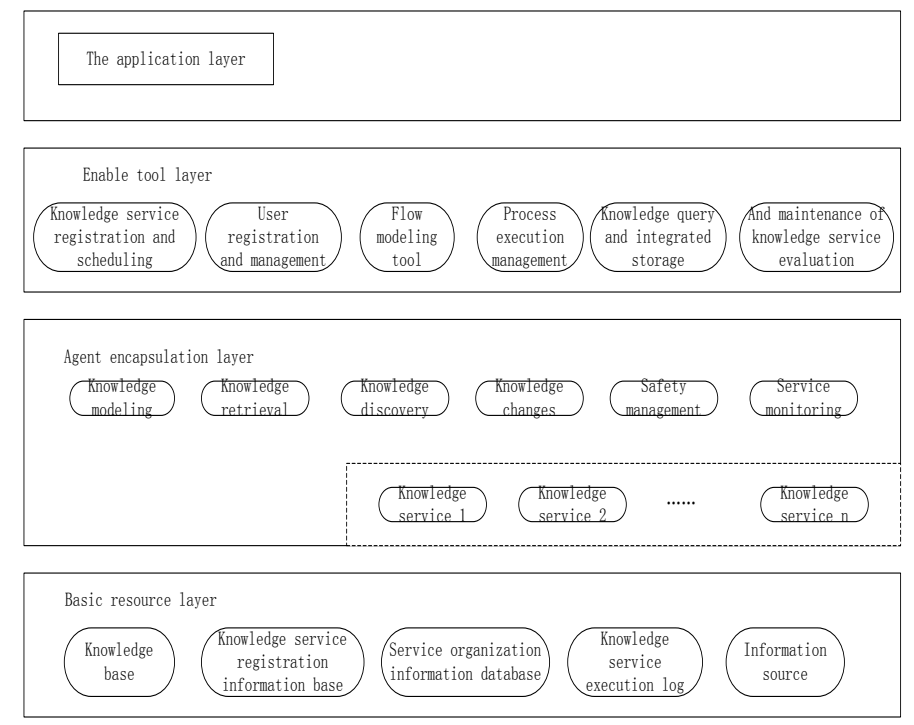

Fig 2 the 4 layers of the platform

The distributed loosely coupled service platform uses the existing network technology can be directly docking service components of various packaging good, its biggest feature is that the web service and maintenance can be independent, so this method reduces the difficulty of platform development flexibility and the scalability of the system is improved, as shown in fig 3. 


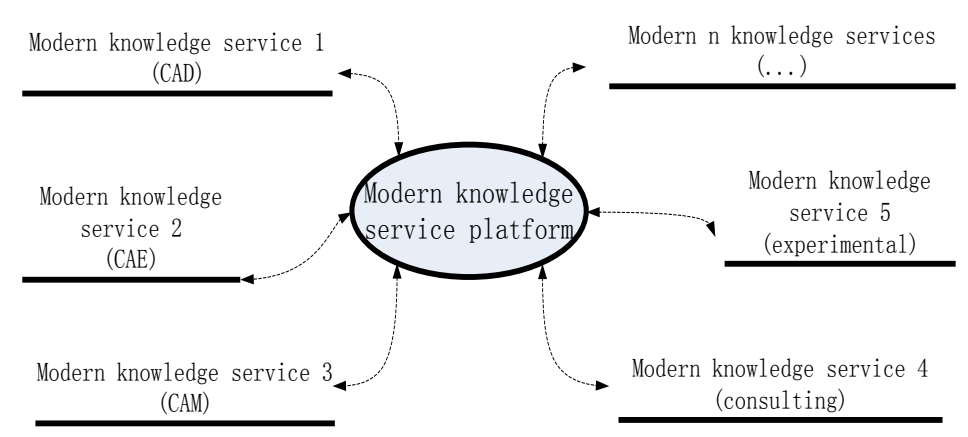

Fig3 Modern knowledge service platform

According to the construction process of knowledge service network technology mode can be constructed the knowledge service of corresponding components in the theoretical research, the numerical simulation, the experimental research and the engineering validation. [3]Through the support of the component integration the product design work is finished.

\section{Application framework and industry characteristics}

Mechanical engineering as a traditional engineering discipline, compared with other professions, has the following features:

a) Design and manufacture process of the products is a process of intensive and knowledge intensive experience. The process of its accumulated experience is particularly important.

b) Product diversification, serialization, variety, focuses on technological innovation, upgrading faster way to manage the implementation of batch management products.

c) Production mode diversification, have large, medium and small batch production mode, including assembly technology as the core.

d) Around the customer order-based production methods.

e) Special equipment and general equipment arrangement as whole, specialized equipment closely related to the quality of the products, the use of special equipment and management have higher requirements.

f) Manufacturing process adopted by the method, equipment, processing technology, etc. are closely related to the production process has contact.

g) The higher the degree of information technology in the manufacturing process, the more likely the emergence of unbalanced data, the information cannot be re-used and shared retardation and other information.

In the mechanical engineering company, in order to reuse the knowledge management and management effectively, facilitate the information system of information sharing and interoperability between heterogeneous systems, according to the characteristics of the industry, refer Knowledge ontology application in other areas.

Application layer can contain any knowledge of the system, such as: CAX systems, product configuration management, product modeling, collaborative manufacturing and collaborative product development. Mechanical engineering ontology in accordance with a certain proportion of particle size is established, such as: machining, process, tools, extra bed, business processes, product design, enterprise and workshop etc. establish the ontology in the field of machinery. According to the actual needs, establish more detailed granularity ontology through ontology integration and mapping of the ontology to form a fine-grained bulk coarse-grained. Coarse-grained ontology gradually forms knowledge base of application system, using the shared reasoning technology, and realized the application of knowledge. [4-5]

\section{The knowledge flow in knowledge service platform}

In the design process of the knowledge innovation platform, how to acquire knowledge and its flow pattern and gradually applied to the product development process is described in the following article. 


\section{Macroscopic knowledge flow}

In the analysis of macroscopic knowledge flow pattern, the focus is on the integration of existing knowledge and the product performance of design knowledge sources. The integrated cyber source integration strategy is putted forward. The key problem is that in the distributed resource environment highly dispersed nature of cyber source is recombined. [6] Through the development and implementation of integrated resource engine and resource pool, the knowledge net is formed in order to facilitate the users from the network knowledge island to requiring knowledge and locating the required knowledge. Using the UDDI resource registry and the other systems' integration, the modern design knowledge service platform is built with the resource unit service project.

The purpose of modern knowledge service platform design is to promote China's manufacturing enterprises to improve product design competition in the products of independent innovation, therefore it should be the overall planning from the user perspective. [7]On the above understanding, hierarchical operation mechanism was studied on the platform of various coupling knowledge flow. Knowledge flow in the corresponding topological relations is shown in Figure 6. In the platform of knowledge flow includes not only the interaction flow between parallel units but also includes the mutual flow unit. It is a kind flow of distributed integrated knowledge.

\section{Micro knowledge flow}

In the study of knowledge flow process, the knowledge field effect and the knowledge agglomeration effect occur, we analysis the dynamic effect of knowledge flow in the process of knowledge flow. Knowledge flow is carried out in the knowledge field effect. In this process it will involve the entity as the dynamic knowledge absorption and accept the tasks related to the field. In the comprehensive effect of various related knowledge unit and the environmental factors, the tasks is clear to be optimized and be solved. After the completion the final solution comes out. [8-9]

Carries on the elaboration to the knowledge flow process from the energy point of view, the basic rules of knowledge flow are that the knowledge always flows from the high energy to the low energy, the total energy flow process remain unchanged. [10]Involving the single knowledge energy model of the knowledge flow process unit is shown in the following formula. According to the specific object of knowledge, the potential difference of knowledge A unit and knowledge $\mathrm{B}$ unit is the intellectual potential $\Delta U$. The single knowledge energy model as the basic unit can be extended, the whole process of the complex knowledge flow can be modeled and analyzed.

The energy dynamic balance in the system of A and B:

$$
U_{\mathrm{a}}=U_{b}+E_{a b}+E_{d}
$$

The knowledge flow process can be divided into two kinds of situations: totally knowledge transitive, $U_{a}=U_{b}, \Delta U=0$.This is an ideal state, it is good for a full flow and communication. Knowledge incomplete pass, $U_{a}>U_{b} \Delta U=0$. This is generally caused by blocked, it's harm for the spread and the full use of knowledge.

For $E_{\mathrm{d}}$, it is not related to the environmental factors, knowledge providers and knowledge receiver's own factors, it is a dynamic process:

$$
E_{\mathrm{d}}=E_{\mathrm{d}}\left(e, f_{a}, f_{b}, t\right)
$$

Type: e represents environment; $f_{a}$ represents the knowledge dissipation effect of the knowledge

provider, $f_{b}$ represents the knowledge dissipation effect of the knowledge receivers, $\mathrm{t}$ represents the time.

\section{Conclusion}

This paper constructs a solution which can support the whole life cycle of product innovation design in the architecture model of knowledge service platform. From the macroscopic points, we proposed an integrated cyber source integration strategy and also combined with the example that has conducted the research to the hierarchical operation mechanism of knowledge flow in the 
various coupling platform. From the micro level in the energy perspective we try to reveal the nature of knowledge flow law and analysis of the effect of knowledge and the agglomeration field.

The design process involves many fields of knowledge and the design process need to call other disciplines and fields, so the modern design does not preclude other design theory and method, which belongs to an extensible theory. The integration of existing design resources, design theory and methods to better is a challenge in this field, but also that improving the competitiveness of products to solve the problem of our country in the present is the key problem in the world. Therefore, this paper presents the modern design knowledge service platform and the knowledge flow are analysed, but the future research work need to do more and more work to promote the efficiency and quality of our product design.

\section{References}

[1] DONALD WM, WILLIAM MP. Aerospace systems design in NASA's collaborative engineering environment[j]. Acta Astroonautica, 2000, 47 (5):255-264.

[2] TANER B, MARK SF. A framework for design in the large [EB/OL].

[3] FOSTER I, ESSELMAN C, NICK J M, et al. The physiology of the grid services architcture for distributed systems integration [EB/OL].

[4] Committee on Advanced Engineering Environment, et al. Advanced engineering environment: Phase 1. Achieving the vision [M]. Washington, D.C: National Academy Press, 1999.

[5] Martens D, Backer M D, Haesen R, et al.Ant-based approach to the knowledge fusion [M]//Lecture Notes in Computer science. Heidilberg: Springer, 2006, 4150:84-05.

[6] Shave M J R. Ontological structures for knowledge sharing [J].New Review of Information Networking, 1997, 24(3):125-133.

[7] NECHES R, FIKERS R, FININ T, et al.Enabling technology for knowledge sharing [J]. AI Magazine, 1991, 12(3):36-56.

[8] EHRIG M, SURE Y. Ontology mapping-an integrated approach[R]. Karlsruhe, Germany: University of Karlsruhe, 2004.

[9] SUDARSAN R, FENVES SJ, SRIRAM R D, et al. A product information modeling framework for product lifecycle management [J]. Computer-Aided Design, 2005, 37(13): 1399-1411.

[10] DOMINGUE J, MOTTA E, GARCIA O C. Knowledge modelling in webonto and OCML; a user guide. http:/ /kmi.open. ac.uk/projects /webonto /user_guide, 2.4. pdf. 